\title{
METHOD OF INFRARED SPECTRA REGISTRATION OF ACTIVATED CARBONS IN POTASSIUM BROMIDE PELLETS
}

\author{
Diana Shepel $^{\mathrm{a}^{*}}$, Tatiana Goreacioc ${ }^{\mathrm{a}, \mathrm{b}}$, Tudor Lupascu ${ }^{\mathrm{a}}$, Mihail Filippov $^{\mathrm{a}}$, Maria Rusu ${ }^{\mathrm{a}}$ \\ anstitute of Chemistry of Academy of Science of Moldova, 3, Academiei str., Chisinau MD-2028, Republic of Moldova \\ ${ }^{b}$ Institute of Ecology and Geography of Academy of Sciences of Moldova, 1, Academiei str., \\ Chisinau MD-2028, Republic of Moldova \\ "email: dianashepel@gmail.com
}

\begin{abstract}
This communication is devoted to the elaboration of a new optimal technique of infrared spectra registration of activated carbons in potassium bromide pellets. Authors investigated the dependence of the intensity of the least overlapping infrared bands of activated carbons on the conditions of preparation, recording of the spectrum, and the degree of homogenization with $\mathrm{KBr}$.
\end{abstract}

Keywords: infrared spectroscopy, activated carbons, potassium bromide $\mathrm{KBr}$ pellets.

Received: February 2015/Revised final: March 2015/ Accepted: March 2015

\section{Introduction}

Infrared (IR) spectroscopy has an important role in the study of the active carbon structure. In the study of carbons, research of pressed pellets with alkali metal halides is used for this purpose, as well as IR spectroscopy of attenuated total reflectance (ATR). Tabletting method has become the most popular method of obtaining IR spectra of active carbons [1-3]. However, errors may occur in the measurement of the intensity of the bands due to the inhomogeneous distribution of the sample in the pellet. When the sample is distributed unevenly in the pellet, the beam of the Fourier Transform Infrared (FTIR) spectrometer can fall on pure $\mathrm{KBr}$ regions with no test substance and this may lead to distortion of the measured intensities of the bands. The increase of voids caused by the inhomogeneous distribution of the test substance in the pellet leads to the decrease of recorded bands intensities and stronger bands decrease more significantly than weaker ones. Thus the so-called smoothing of the spectrum occurs.

The aim of this work was to study the dependence of the intensity of the least overlapping infrared bands of activated carbons on the conditions of preparation, recording of the spectrum, the degree of homogenization with $\mathrm{KBr}$, as well as the elaboration of an optimal method of sample preparation as a pellet of active carbon homogenate with $\mathrm{KBr}$ for IR spectrum registration.

\section{Experimental}

The method of IR spectra registration of activated carbons in $\mathrm{KBr}$ pellets.

Amount of 0.40-0.50 mg of activated carbon was homogenized in an agate mortar with $300 \mathrm{mg}$ of optically pure $\mathrm{KBr}$, vacuumized for $5 \mathrm{~min}$ and pressed under $10 \mathrm{ton} / \mathrm{cm}^{2}$ for $15 \mathrm{~min}$. The area $\left(\mathrm{S}, \mathrm{cm}^{2}\right)$ of the $\mathrm{KBr}$ pellet was $1.13 \mathrm{~cm}^{2}$ (Zeiss standard). A light grey transparent pellet without visually noticeable grains was obtained. IR spectra were recorded on a PerkimElmer FTIR Spectrometer Spectrum 100, (2007) in the wave number range of $4000-400 \mathrm{~cm}^{-1}$. Prior to the preparation of pellets and spectral analysis, the samples of carbons and $\mathrm{KBr}$ were dried and stored above anhydrous silica gel in an exicator. The optimal conditions of spectra recording were as follows: spectral resolution $-2 \mathrm{~cm}^{-1}$, the scan number - 10, the number of automatic spectrum smooth -2 . Spectral data handling and baseline correction was carried out by the Spectrum software Version 6.2.0.0055 (2007). After the first registration of the spectrum the pellet was transferred quantitatively into a mortar and triturated (homogenized). The powder was repressed and IR spectrum was rerecorded. The operations of homogenization and IR spectrum registration were repeated until maximal stable values of absorption (A) were obtained. The IR spectrum was recorded using as control the same pellet made from pure ground potassium bromide.

Effects of various factors during the IR spectra registration in the $\mathrm{KBr}$ pellets have been examined on the example of a modified activated carbon AG-5ox (Table 1) obtained from a commercial sample of carbon AG-5. Activated carbon AG-5 was produced in granulated form from coal dust (coals mixture) and an adhesion agent by steam treatment at 850 - $950{ }^{\circ} \mathrm{C}$ [4]. Sample of AG-5ox has been prepared by oxidation of AG-5 with concentrated nitric acid as described in [5]. $0.480 \mathrm{mg}$ of the active carbon sample AG-5ox was taken to prepare the $\mathrm{KBr}$ pellet according to the above-mentioned method. The concentration of AG-5ox was $\mathrm{C}=0.425 \mathrm{mg} / \mathrm{cm}^{2}$ and was held constant during the experiment.

\section{Results and discussion}

This communication described the method of sample preparation in the form of homogenate pellets of active carbon with $\mathrm{KBr}$ for recording the IR spectrum. The dependence of the intensity of the least overlapping infrared bands of activated carbons on the conditions of preparation, recording of the spectrum, and the degree of homogenization with potassium bromide has been investigated. 
The recorded intensities of the IR bands depend on several factors:

1. Dependence on particle sizes. With all other conditions maintained equal, light scattering increases with the particle size in comparison with the wavelength. The particle size is comparable to the wavelength of the absorbed light in the ideal case. In the spectral region $4000-400 \mathrm{~cm}^{-1}$, particle size $2.5-25$ microns and particle size greater than 2.5 microns, the scattering of light decreases with increasing wavelength. The baseline correction method was used to compensate for the scattered light effect, consisting in the measurement of light absorption relative to a line drawn through the minima of the absorption (A) spectrum, respectively, through the transmittance (T,\%) maxima.

2. Dependence on the uniformity of particle distribution in the $\mathrm{KBr}$ pellets. The recorded intensity of bands (A) depends not only on particle size but also on the equal distribution in the pellet. In the case of an uneven distribution of particles in the pellet, voids consisting of the pure $\mathrm{KBr}$ are formed between particles of analyzed sample, i.e. defects observed in the continuous distribution of particles. The increase in defects leads to the decrease of measured bands intensities, and this decrease is proportional to band intensity. The result is a so-called smoothing of the spectrum $[6,7]$. We have used techniques of consecutive sample homogenization with $\mathrm{KBr}$ to eliminate this defect. The pressed pellet has been reground and repressed consecutively several times to achieve constancy of the measured value A of the bands of varying intensity.

3. Homogenization of the mixture of investigated sample and $\mathrm{KBr}$ in an open atmosphere leads to moisture sorption. The bands of water absorbed by $\mathrm{KBr}$ appear in the spectrum $v(\mathrm{OH})$ at $3450 \mathrm{~cm}^{-1}$ and $\delta(\mathrm{HOH})$ at $1630 \mathrm{~cm}^{-1}[1,8]$. This absorption is compensated by the introduction of the control pellet of pure $\mathrm{KBr}$ in the reference beam of the spectrometer.

4. The measured value of absorption A depends on the weight of analyzed substance (g, mg) that is uniformly distributed over the area of the pellet $\left(\mathrm{S}, \mathrm{cm}^{2}\right)$.

At a constant area of the pellet, any variation in the amount of $\mathrm{KBr}$ in the homogenate only leads to a change in the thickness of the pellet, but the quantity of the analyte projected in the beam onto the slit of the instrument is not changed. Thus the classic formula of the Lambert-Beer law for solid substance homogenized in the pellet with $\mathrm{KBr}$ has the form $K=A / C$. The $\mathrm{K}$ values (Eqs.(1) and (2)) of the intensities of the bands in the spectrum were compared.

$C=g / S$,

$K=\frac{A \cdot S}{g}, \mathrm{~cm}^{2} \times \mathrm{mg}^{-1}$,

where A - measured absorbance (optical density); S - area of the pellet, $\mathrm{cm}^{2} ; \mathrm{g}$ - weight of the sample in the pellet, mg.

In order to compensate water absorption by the pellet during homogenization, the control pellet made from pure $\mathrm{KB}$ was placed in the beam of the FTIR spectrometer. The Carl Zeiss Company recommended a weight $300 \mathrm{mg}$ of $\mathrm{KBr}$ on a standard area $1.13 \mathrm{~cm}^{2}$ of a branded pellet to obtain sufficient firmness.

The results of investigations of the influence of various factors on the registration of the IR spectra of the modified activated carbon AG-5ox in $\mathrm{KBr}$ pellets are shown in Table 1.

Table 1

The dependence of optical density (A) of the least overlapping bands on the conditions of preparation and registration of the IR spectrum of the sample AG-5ox.

\begin{tabular}{lcccccc}
\hline \multirow{2}{*}{ Conditions of spectrum registration } & \multicolumn{3}{c}{ Band $_{1}$} & \multicolumn{2}{c}{ Band $_{2}$} & \multicolumn{2}{c}{ Band $_{3}$} \\
\cline { 2 - 7 } & $v_{1}, \mathrm{~cm}^{-1}$ & $A_{1} \cdot 10^{3}$ & $v_{2}, \mathrm{~cm}^{-1}$ & $A_{2} \cdot 10^{3}$ & $v_{3}, \mathrm{~cm}^{-1}$ & $A_{3} \cdot 10^{3}$ \\
\hline Immediately after pressing & 1711 & 415 & 1561 & 433 & 1101 & 457 \\
In 20 hours & 1717 & 428 & 1559 & 451 & 1096 & 474 \\
Rotation of the pellet by $90^{\circ}$ & 1717 & 430 & 1560 & 454 & 1096 & 477 \\
\hline
\end{tabular}

It was found that rotation of the pellet in the beam of the instrument didn't produce any changes. Consequently, the sample was uniformly distributed in the pellet. The spectrum of the sample in the pellet did not change after 20 hours, showing there was no interaction of the pressed AG-5ox with $\mathrm{KBr}$ during that time. No changes in the spectrum were observed after reapplying pressure on the uncrushed pellet during 3 hours.

The sample of AG-5ox was also used to investigate the dependence of the evenness of its distribution on the degree of homogenization in the $\mathrm{KBr}$ pellet (Table 2, Figure 1). After the first registration of the IR spectrum the pellet was subjected to repeated homogenization in a mortar, and another repressing. Pellet trituration in a mortar and pressing were repeated until stable values of absorption (A) were obtained.

Figure 1 shows the spectra of the AG-5ox sample as function of the degree of homogenization. Three least overlapping bands $1713(\mathrm{C}=\mathrm{O}), 1560(\mathrm{C}=\mathrm{C}), 1098(\mathrm{C}-\mathrm{C}) \mathrm{cm}^{-1}$ were chosen for quantitative characterization of the changes in the spectrum of AG-5ox during sample homogenization (Table 2). The first and second homogenizations 
were insufficient to obtain a deeply embossed spectrum. The maximum and constant values of the IR bands intensities were achieved only after the third and fourth homogenizations. The increase in the homogenization degree made the spectra more relief and the latter reached a limit, which corresponded to previously reported data [1, 2, 6-8].

Table 2

The dependence of the IR bands intensities of modified activated carbon AG-5ox on the degree of homogenization.

\begin{tabular}{ccccccc}
\hline \multirow{2}{*}{ Degree of homogenization } & \multicolumn{2}{c}{ Band $_{1}$} & \multicolumn{2}{c}{ Band $_{2}$} & \multicolumn{2}{c}{ Band $_{3}$} \\
\cline { 2 - 7 } & $v_{1}, \mathrm{~cm}^{-1}$ & $A_{1} \cdot 10^{3}$ & $v_{2}, \mathrm{~cm}^{-1}$ & $A_{2} \cdot 10^{3}$ & $v_{3}, \mathrm{~cm}^{-1}$ & $A_{3} \cdot 10^{3}$ \\
\hline 1 & 1719 & 424 & 1567 & 449 & 1098 & 474 \\
2 & 1714 & 464 & 1548 & 485 & 1097 & 519 \\
3 & 1713 & 482 & 1548 & 514 & 1098 & 560 \\
4 & 1713 & 489 & 1560 & 526 & 1099 & 574 \\
\hline
\end{tabular}

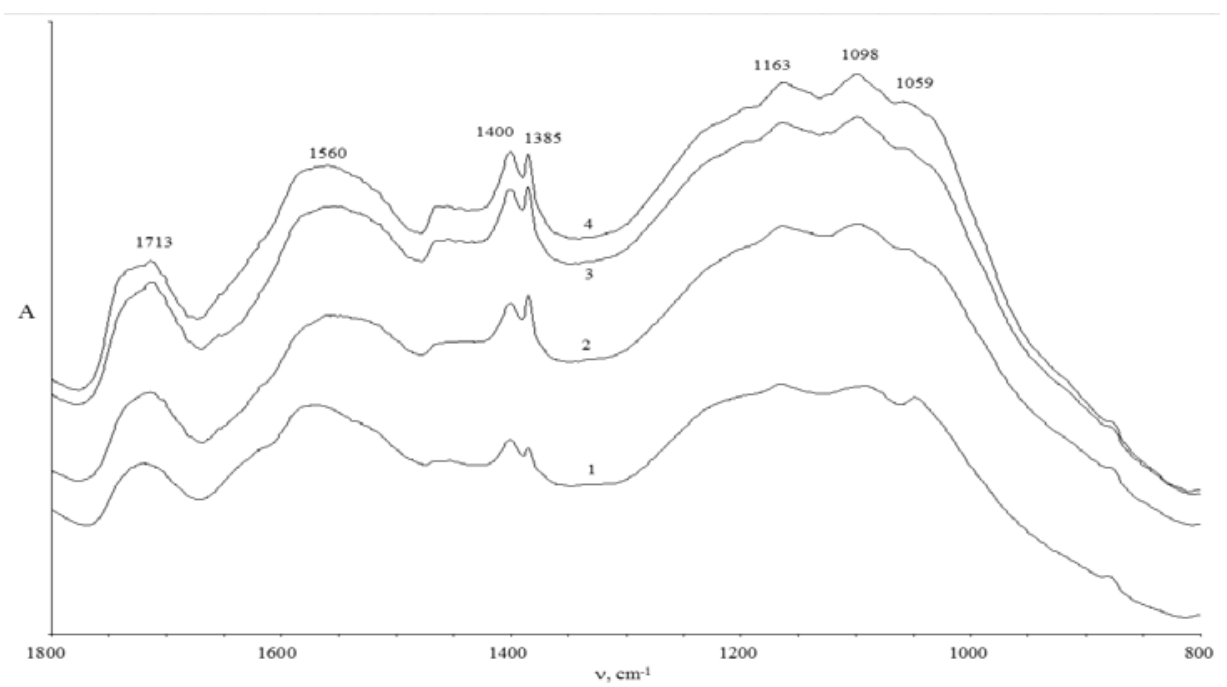

Figure 1. IR spectra of the sample AG-5ox as function of the degree of homogenization with KBr. The spectrum number corresponds to the degree of homogenization.

\section{Conclusions}

The investigation of the dependence of the optical density of the least overlapping bands in the IR spectrum of the activated carbon on the conditions of preparation and registration of the spectrum showed that the pressed sample of activated carbon was distributed evenly in the pellet and didn't suffer any changes after homogenization with $\mathrm{KBr}$. An optimum technique for IR spectra registration of activated carbons in the $\mathrm{KBr}$ pellets has been proposed. The maximum and constant values of the intensities of the IR bands were achieved after the third and fourth homogenization of the $\mathrm{KBr}$ pellets.

\section{References}

1. Applied Infrared Spectroscopy. Edited by Kendall, D.N. Mir: Moscow, 1970, 376 p. (in Russian).

2. Roop, C. B.; Meenakshi, G. Activated Carbon Adsorption. A CRC Press Book. Taylor\&Francis Group: New-York, 2005, pp. 22-34.

3. Cuhadaroglu, D.; Uygun, O.A. Production and characterization of activated carbon from a bituminous coal by chemical activation. African Journal of Biotechnology, 2008, 7(20), pp. 3703-3710.

4. GOST 20777-75. Granulated active carbon AG-5 (in Russian).

5. Goreacioc T. Oxidation and characterization of active carbon AG-5. Chemistry Journal of Moldova, 2015, 10(1), pp. 75-82.

6. Elliot, A. Infrared spectra and structure of polymers. Mir: Moscow, 1972, 160 p. (in Russian).

7. Tremblay, L.; Gagne, J.-P. Fast Quantification of Humic Substances and Organic Matter by Direct Analysis of Sediments Using DRIFT Spectroscopy. Analytical chemistry, 2002, 74(13), pp. 2985-2993.

8. Bellamy, L.J. The Infrared Spectra of Complex Molecules. Foreign Literature Publishing House: Moscow, 1963, 592 p. (in Russian). 